\title{
Teletransporte Quântico, Dinâmica de Campos Térmicos e a Álgebra de Lie $s u(2)$
}

Quantum Teleportation, Thermofield Dynamics and the Lie Algebra su(2)

\author{
M.A.S. Trindade* \\ Departamento de Ciências Exatas - UNEB \\ R. Silveira Martins, 2555, Cabula, Salvador - Bahia - 41150-000
}

S. Floquet

CCIVIL Campus Juazeiro - UNIVASF

Av. Antônio C. Magalhães, 510,

Country Club, Juazeiro - BA - 48902-300

\author{
J.D.M. Viannat \\ Instituto de Física - UFBA \\ Campus Universitário de Ondina, \\ Salvador - Bahia - 40210-340
}

\begin{abstract}
Neste trabalho investigamos a relação entre a duplicação do formalismo da Dinâmica de Campos Térmicos (DCT) e o procedimento de purificação na teoria da Informação Quântica. Em seguida, analisamos efeitos de temperatura no teletransporte quântico de estados bosônicos, utilizando a DCT, e através da álgebra de Lie $s u(2)$ termalizada realizamos o protocolo do teletransporte. Derivamos o operador densidade associado aos estados termalizados a fim de calcularmos a fidelidade do teletransporte e o limite de temperatura nula.
\end{abstract}

Palavras-chaves: Teletransporte Quântico; Dinâmica de Campos Térmicos; Álgebra de Lie $s u(2)$.

In this work we explore the relationship between the duplication of the Thermal Field Dynamics (TFD) formalism and the purification procedure in Quantum Information Theory. Then, we analyze temperature effects in the quantum teleportation of bosonic states, using the TFD, and through the thermalized Lie algebra $s u(2)$ we perform the teleportation protocol. We derive the density operator associated with the thermal states in order to calculate the teleportation fidelity and the zero temperature limit.

Key-words: Quantum Teleportation; Thermofield Dynamics; Lie Algebra $s u(2)$.

\section{INTRODUÇÃO}

Na teoria da computação quântica, as unidades básicas de informação são os q-bits. $\mathrm{O}$ estado geral de um q-bit é descrito pela superposição dos estados $|0\rangle$ e $|1\rangle$,

$$
|\psi\rangle=a|0\rangle+b|1\rangle,
$$

\footnotetext{
*Endereço Eletrônico: matrindade@uneb.br

${ }^{\dagger}$ Endereço Permanente: Instituto de Física - UnB, Brasília-DF, 70919-970.
}

onde $a, b \in \mathbb{C}$ e $|a|^{2}+|b|^{2}=1$. Tal conjunto $\{|0\rangle,|1\rangle\}$ constitui uma base para um espaço de Hilbert bidimensional, cuja representação matricial consiste em,

$$
|0\rangle \leftrightarrow\left(\begin{array}{l}
1 \\
0
\end{array}\right), \quad|1\rangle \leftrightarrow\left(\begin{array}{l}
0 \\
1
\end{array}\right)
$$

As operações sobre os q-bits são representadas por portas lógicas quânticas, que são associadas a matrizes unitárias, tais como as matrizes de 
Pauli,

$$
X=\left(\begin{array}{ll}
0 & 1 \\
1 & 0
\end{array}\right), Y=\left(\begin{array}{cc}
0 & -i \\
i & 0
\end{array}\right), Z=\left(\begin{array}{cc}
1 & 0 \\
0 & -1
\end{array}\right) .
$$

de Hilbert é expandido pelos vetores,

$$
\{|00\rangle,|01\rangle,|10\rangle,|11\rangle\}
$$

cuja representação matricial consiste em,

Para um sistema com dois q-bits, o espaço

$$
|00\rangle \leftrightarrow\left(\begin{array}{l}
1 \\
0 \\
0 \\
0
\end{array}\right),|01\rangle \leftrightarrow\left(\begin{array}{l}
0 \\
1 \\
0 \\
0
\end{array}\right),|10\rangle \leftrightarrow\left(\begin{array}{l}
0 \\
0 \\
1 \\
0
\end{array}\right),|11\rangle \leftrightarrow\left(\begin{array}{l}
0 \\
0 \\
0 \\
1
\end{array}\right)
$$

Podemos notar a existência de estados, pertencentes ao sistema composto por dois q-bits, para os quais $|\psi\rangle$ não pode ser escrito como $\left|\psi_{A}\right\rangle \otimes\left|\psi_{B}\right\rangle$, em que os índices $A$ e $B$ reportamse aos subsistemas $A$ e $B$, respectivamente.

Estados que possuem está característica são ditos emaranhados, e os estados de Bell são exemplos paradigmáticos:

$$
\begin{aligned}
& \left|\Phi^{+}\right\rangle=\frac{1}{\sqrt{2}}(|00\rangle+|11\rangle), \\
& \left|\Psi^{+}\right\rangle=\frac{1}{\sqrt{2}}(|01\rangle+|10\rangle), \\
& \left|\Phi^{-}\right\rangle=\frac{1}{\sqrt{2}}(|00\rangle-|11\rangle), \\
& \left|\Psi^{-}\right\rangle=\frac{1}{\sqrt{2}}(|01\rangle-|10\rangle) .
\end{aligned}
$$

Os estados de Bell podem ser gerados a partir da aplicação de duas portas lógicas, conhecidas como Hadamard $(H)$ e NãoControlado (CNOT), a partir do circuito quântico ilustrado na figura 1 .

A representação matricial da porta lógica $H$,

$H=\frac{1}{\sqrt{2}}\left(\begin{array}{ll}1 & 1 \\ 1 & -1\end{array}\right) ; \begin{aligned} & H|0\rangle=\frac{1}{\sqrt{2}}(|0\rangle+|1\rangle) \\ & H|1\rangle=\frac{1}{\sqrt{2}}(|0\rangle-|1\rangle),\end{aligned}$

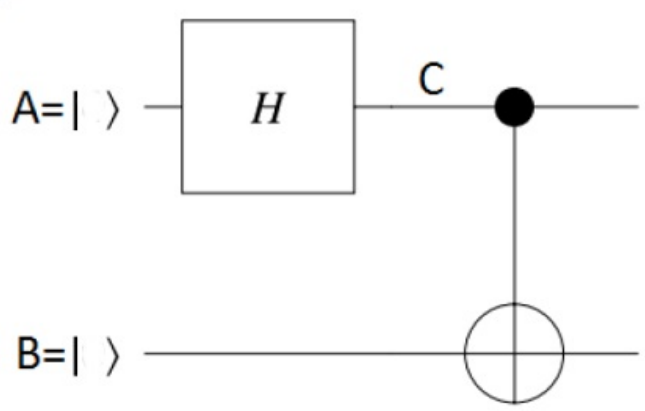

FIGURA 1: Circuito quântico para geração dos estados de Bell.

Fonte: Os autores.

e da porta lógica $C N O T$,

CNOT $=\left(\begin{array}{llll}1 & 0 & 0 & 0 \\ 0 & 1 & 0 & 0 \\ 0 & 0 & 0 & 1 \\ 0 & 0 & 1 & 0\end{array}\right) ; \begin{aligned} & C N O T|00\rangle=|00\rangle \\ & C N O T|01\rangle=|01\rangle \\ & C N O T|10\rangle=|11\rangle \\ & C N O T|11\rangle=|10\rangle\end{aligned}$

permite escrever os estados de Bell da forma,

$$
\begin{aligned}
\text { CNOT } H_{A}|00\rangle & =\operatorname{CNOT}\left[\frac{1}{\sqrt{2}}(|0\rangle+|1\rangle)|0\rangle\right] \\
& =\frac{1}{\sqrt{2}}(|00\rangle+|11\rangle)=\left|\Phi^{+}\right\rangle, \\
\text {CNOT } H_{A}|01\rangle & =\operatorname{CNOT}\left[\frac{1}{\sqrt{2}}(|0\rangle+|1\rangle)|1\rangle\right] \\
& =\frac{1}{\sqrt{2}}(|01\rangle+|10\rangle)=\left|\Psi^{+}\right\rangle,
\end{aligned}
$$




$$
\begin{aligned}
\text { CNOT } H_{A}|10\rangle & =\text { CNOT }\left[\frac{1}{\sqrt{2}}(|0\rangle-|1\rangle)|0\rangle\right] \\
& =\frac{1}{\sqrt{2}}(|00\rangle-|11\rangle)=\left|\Phi^{-}\right\rangle, \\
\text {CNOT } H_{A}|11\rangle & =\text { CNOT }\left[\frac{1}{\sqrt{2}}(|0\rangle-|1\rangle)|1\rangle\right] \\
& =\frac{1}{\sqrt{2}}(|01\rangle-|10\rangle)=\left|\Psi^{-}\right\rangle,
\end{aligned}
$$

onde o subíndice $A$ reforça que a porta lógica Hadamard só atua no primeiro subsistema.

Existem critérios de separabilidade, capazes de distinguir se um estado puro bipartite, dado por,

$$
|\psi\rangle=\sum_{j, k} a_{j k}|j\rangle|k\rangle,
$$

em que $|j\rangle$ e $|k\rangle$ correspondem a bases ortonormais dos dois subsistemas $A$ e $B$, é fatorável. Um critério bastante empregado é a decomposição de Schmidt [1], que afirma que todo estado puro bipartite pode ser escrito como uma soma de termos biortogonais,

$$
|\psi\rangle=\sum_{i} \lambda_{i}\left|i_{A}\right\rangle\left|i_{B}\right\rangle
$$

onde as bases $\left|i_{A}\right\rangle$ e $\left|i_{B}\right\rangle$ são denominadas bases de Schmidt e o número de autovalores $\lambda_{i}$ (coeficientes de Schmidt) é denominado número de Schmidt. Pode-se perceber que um estado puro é fatorável se, e somente se, seu número de Schmidt vale 1. Os subsistemas $A$ e $B$ não possuem necessariamente a mesma dimensão e esse resultado, em princípio, pode ser generalizado para um número arbitrário de subsistemas [1]. Os estados emaranhados, que não são fatoráveis, são fundamentais para o teletransporte quântico [19].

Podemos definir o teletransporte quântico como um fenômeno onde ocorre a transferência da informação quântica, o que possibilita a reconstrução da informação contida num estado, de um sistema quântico A, para um outro sistema quântico B, a partir da aniquilação do estado de interesse. O primeiro protocolo de teletransporte quântico foi proposto por Ben- net [2], em 1993, e a primeira realização experimental ocorreu em 1998 [3].

Nosso objetivo aqui é investigar o efeito que a temperatura pode causar nestes estados, utilizando o formalismo da dinâmica de campos térmicos. Um investigação nesse sentido já foi realizada na referência [4, 5]; entretanto nossa proposta é a de utilização de estados quânticos mais realísticos [5], envolvendo a chamada representação duplo-trilho [6]. Para tal intento, necessitamos generalizar expressões conhecidas da dinâmica de campos térmicos, em que apenas um modo do campo é considerado. Em suma, o que propomos é considerar a possibilidade de teletransporte de estados térmicos e tratá-los como estados puros seguindo a prescrição da DCT [4, 5, 7].

Na seção II], realizaremos uma revisão da dinâmica de campos térmicos nos restringindo aos aspectos fulcrais para a compreensão dos cálculos realizados, apresentando a termalização do oscilador harmônico bosônico, dos operadores de criação e aniquilação e do operador densidade termalizado. Na seção III, realizamos uma analogia entre a purificação e o formalismo DCT. Na seção IV] utilizando a prescrição da DCT, analisamos os efeitos de temperatura no protocolo do teletransporte quântico de estados bosônicos envolvendo a representação duplo-trilho, de interesse da ótica quântica, e a álgebra de Lie termalizada do grupo $S U(2)$, aplicando no cálculo da Fidelidade. Na seção V, apresentamos as conclusões e perspectivas.

\section{DINÂMICA DE CAMPOS TÉRMICOS}

Nesta seção, revisaremos alguns aspectos da DCT fulcrais para o desenvolvimento deste trabalho. Iniciamos analisando um sistema em equilíbrio com um reservatório térmico, em que a média estatística de um observável é dado por,

$$
<A>=\frac{\operatorname{Tr}\left(A e^{-\beta H}\right)}{Z(\beta)}
$$


com $H$ o Hamiltoniano do sistema, $\beta=1 / k_{b} T$, sendo $k_{b}$ a constante de Boltzmann e $T$ a temperatura e $Z(\beta)$ a função de partição, que é dada por $Z(\beta)=\operatorname{Tr}\left(e^{-\beta H}\right)$. Para uma base em termos dos autoestados do Hamiltoniano, $H|n\rangle=E_{n}|n\rangle$, a média estatística do observável é,

$$
<A>=\sum_{n} \frac{e^{-\beta E_{n}}\langle n|A| n\rangle}{Z(\beta)} .
$$

A motivação da DCT é encontrar um estado, $|0(\beta)\rangle$, em que a média estatística de um operador qualquer seja igual a seu valor esperado, ou seja,

$$
\langle 0(\beta)|A| 0(\beta)\rangle:=<A>.
$$

Se supusermos que $|0(\beta)\rangle \in \mathcal{H}$, ao espaço de Hilbert do sistema, encontraremos um absurdo,

$$
|0(\beta)\rangle=\sum_{n}|n\rangle\langle n \mid 0(\beta)\rangle=\sum_{n} g_{n}(\beta)|n\rangle,(14
$$

onde $g_{n}(\beta)=\langle n \mid 0(\beta)\rangle$. Então,

$$
\begin{aligned}
\langle 0(\beta)|A| 0(\beta)\rangle & =\sum_{n, m} g_{n}^{*}(\beta)\langle n|A| m\rangle g_{m}(\beta),(15) \\
& =\sum_{n} \frac{e^{-\beta E_{n}}\langle n|A| n\rangle}{Z(\beta)}
\end{aligned}
$$

e comparando as equações (15) e 16 obtemos,

$$
g_{n}^{*}(\beta) g_{m}(\beta)=\frac{e^{-\beta E_{n}} \delta_{n m}}{Z(\beta)},
$$

que é um absurdo, já que o espaço das funções não tem como satisfazer uma relação de ortogonalidade. Portanto, $|0(\beta)\rangle \notin \mathcal{H}$.

Para que a Eq. (17) seja satisfeita, uma possibilidade é que $g_{n}(\beta)$ seja um vetor de um espaço de Hilbert $\mathcal{H}$, que denotaremos por $\widetilde{\mathcal{H}}$, ou seja, $g_{n}(\beta)=\frac{e^{-\frac{\beta E_{n}}{2}}}{\sqrt{Z(\beta)}}|\widetilde{n}\rangle \in \widetilde{\mathcal{H}}$. Desta forma,

$$
\begin{aligned}
g_{n}^{*}(\beta) g_{m}(\beta) & =\left\langle\widetilde{n}\left|\frac{e^{-\frac{\beta E_{n}}{2}}}{\sqrt{Z(\beta)}} \frac{e^{-\frac{\beta E_{m}}{2}}}{\sqrt{Z(\beta)}}\right| \widetilde{m}\right\rangle \\
& =\frac{e^{-\beta E_{n}} \delta_{n m}}{Z(\beta)},
\end{aligned}
$$

e o vácuo térmico é então denotado por,

$$
|0(\beta)\rangle=\sum_{n} \frac{e^{-\frac{\beta E_{n}}{2}}}{\sqrt{Z(\beta)}}|n, \widetilde{n}\rangle \in \mathcal{H} \otimes \widetilde{\mathcal{H}},
$$

satisfazendo a condição de normalização $\langle 0(\beta) \mid 0(\beta)\rangle=1$.

O sistema termalizado será agora descrito por 2 espaços de Hilbert, $\mathcal{H} \otimes \widetilde{\mathcal{H}}$, sendo $|0, \widetilde{0}\rangle$ o estado de vácuo no espaço duplicado. Uma transformação importante, que associa o estado $|0\rangle \otimes|\widetilde{0}\rangle$ ao vácuo térmico, $|0(\beta)\rangle$, é a transformação de Bogoliubov, definida como,

$$
U(\beta)|0, \widetilde{0}\rangle=|0(\beta)\rangle .
$$

Tendo os elementos básicos necessários para introduzir o formalismo da Dinâmica de Campos Térmicos, trataremos agora dos osciladores Harmônicos Bosônicos, dos Operadores Termalizados e do Operador Densidade Termalizado.

\section{A. Oscilador Harmônico Bosônico Termalizado}

Consideraremos o Hamiltoniano do Oscilador Harmônico Bosônico Unidimensional dado por $H=\omega a^{\dagger} a$, sendo $a$ o operador de aniquilação, $a^{\dagger}$ o operador de criação e $\omega$ a frequência natural do oscilador, onde redefinimos a energia de ponto zero e tomamos $\hbar=$ 1. Para dois modos desacoplados do oscilador harmônico, teremos que o Hamiltoniano é,

$$
H=\omega_{1} a_{1}^{\dagger} a_{1}+\omega_{2} a_{2}^{\dagger} a_{2}
$$

satisfazendo as relação de comutação,

$$
\begin{aligned}
{\left[a_{i}, a_{j}^{\dagger}\right] } & =0, & & i \neq j \\
{\left[a_{i}, a_{j}\right] } & =0=\left[a_{i}^{\dagger}, a_{j}^{\dagger}\right], & & i, j=\{1,2\} \\
{\left[a_{i}, a_{i}^{\dagger}\right] } & =1 & & i=\{1,2\} .
\end{aligned}
$$

Para o oscilador harmônico com dois modos, o espaço de Hilbert do sistema é $\mathcal{H}_{1} \otimes \mathcal{H}_{2}$.

Por construção, o estado térmico $\mid 0(\beta)>\epsilon$ 
$\mathcal{H}_{1} \otimes \mathcal{H}_{2} \otimes \widetilde{\mathcal{H}_{1} \otimes \mathcal{H}_{2}}$ e é,

$$
\begin{aligned}
|0(\beta)\rangle= & \sum_{\substack{n_{1}, n_{2}=0 \\
\\
\times\left|n_{1}, n_{2}, \widetilde{n}_{1}, \widetilde{n}_{2}\right\rangle}}^{+\infty} \frac{e^{-\frac{\beta\left(\omega_{1} n_{1}+\omega_{2} n_{2}\right)}{2}}}{\sqrt{Z(\beta)}} \times
\end{aligned}
$$

$\operatorname{com} Z(\beta)=\frac{1}{1-e^{-\beta \omega_{1}}} \frac{1}{1-e^{-\beta \omega_{2}}}$. Com a introdução do espaço til, surgem os operadores de criação e destruição associados aos dois mo$\operatorname{dos} \widetilde{a}_{1}^{\dagger}, \widetilde{a}_{2}^{\dagger}, \widetilde{a}_{1}$ e $\widetilde{a}_{2}$; esses operadores til satisfazem relações de comutação análogas às 22 . e comutam com qualquer operador sem o til.

O estado térmico pode ser escrito da forma,

$$
\begin{aligned}
|0(\beta)\rangle= & \sqrt{1-e^{-\beta \omega_{1}}} \sqrt{1-e^{-\beta \omega_{2}}} \times \\
& e^{\left(e^{-\frac{\beta \omega_{1}}{2}} a_{1}^{\dagger} \widetilde{a}_{1}^{\dagger}\right)} e^{\left(e^{\left.-\frac{\beta \omega_{2}}{2} a_{2}^{\dagger} \widetilde{a}_{2}^{\dagger}\right)} \times\right.} \\
& \times|0,0, \widetilde{0}, \widetilde{0}\rangle \\
= & e^{-i G_{1}(\beta)} e^{-i G_{2}(\beta)}|0,0, \widetilde{0}, \widetilde{0}\rangle \\
= & e^{-i G(\beta)}|0,0, \widetilde{0}, \widetilde{0}\rangle,
\end{aligned}
$$

sendo $G_{i}(\beta)=-i \theta_{i}(\beta)\left(\widetilde{a}_{i} a_{i}-\widetilde{a}_{i}^{\dagger} a_{i}^{\dagger}\right)$ um operador hermitiano. Por construção a transformação de Bogoliubov é definida por $U(\beta)=$ $e^{-i G(\beta)}$, que leva o vácuo duplicado não térmico no estado térmico $|0(\beta)\rangle$ com,

$$
\begin{aligned}
G(\beta)= & -i \theta_{1}(\beta)\left(\widetilde{a}_{1} a_{1}-\widetilde{a}_{1}^{\dagger} a_{1}^{\dagger}\right)-i \theta_{2}(\beta) \times \\
& \times\left(\widetilde{a}_{2} a_{2}-\widetilde{a}_{2}^{\dagger} a_{2}^{\dagger}\right),
\end{aligned}
$$

tal que,

$$
\begin{aligned}
\cosh \theta_{i}(\beta) & =\frac{1}{\sqrt{1-e^{-\beta \omega_{i}}}}=u_{i}(\beta) \\
\operatorname{senh} \theta_{i}(\beta) & =\frac{e^{-\frac{\beta \omega_{i}}{2}}}{\sqrt{1-e^{-\beta \omega_{i}}}}=v_{i}(\beta),
\end{aligned}
$$

são as funções cosseno e seno hiperbólicas.

\section{B. Operadores Termalizados}

Assim como estados térmicos, é importante definir operadores termalizados. Tais operadores termalizados podem ser introduzidos como transformações de similaridade da transformação de Bougoliubov,

$$
O(\beta)=U(\beta) O U^{\dagger}(\beta)
$$

em que $O$ é um operador não-termalizado. Sendo assim, os operadores de criação e aniquilação térmicos são:

$$
\begin{aligned}
a_{i}^{\dagger}(\beta) & =U(\beta) a_{i}^{\dagger} U^{\dagger}(\beta), \\
a_{i}(\beta) & =U(\beta) a_{i} U^{\dagger}(\beta), \\
\widetilde{a}_{i}^{\dagger}(\beta) & =U(\beta) \widetilde{a}_{i}^{\dagger} U^{\dagger}(\beta), \\
\widetilde{a}_{i}(\beta) & =U(\beta) \widetilde{a}_{i} U^{\dagger}(\beta),
\end{aligned}
$$

Utilizando a relação $e^{-i B} A e^{i B}=$ $A+(-i)[B, A]+\frac{(-i)^{2}}{2 !}[B,[B, A]]+$ $\frac{(-i)^{3}}{3 !}[B,[B,[B, A]]]+\ldots$, podemos escrever as relações (27) da forma,

$$
\begin{gathered}
\left\{\begin{array}{l}
a_{i}(\beta)=u_{i}(\beta) a_{i}-v_{i}(\beta) \widetilde{a}_{i}^{\dagger} \\
a_{i}^{\dagger}(\beta)=u_{i}(\beta) a_{i}^{\dagger}-v_{i}(\beta) \widetilde{a}_{i} \\
\widetilde{a}_{i}(\beta)=u_{i}(\beta) \widetilde{a}_{i}-v_{i}(\beta) a_{i}^{\dagger} \\
\widetilde{a}_{i}^{\dagger}(\beta)=u_{i}(\beta) \widetilde{a}_{i}^{\dagger}-v_{i}(\beta) a_{i}
\end{array}\right. \\
\left\{\begin{array}{l}
a_{i}=u(\beta) a_{i}(\beta)+v(\beta) \widetilde{a}_{i}^{\dagger}(\beta) \\
a_{i}^{\dagger}=u(\beta) a_{i}^{\dagger}(\beta)+v(\beta) \widetilde{a}_{i}(\beta) \\
\widetilde{a}_{i}=u(\beta) \widetilde{a}_{i}(\beta)+v(\beta) a_{i}^{\dagger}(\beta) \\
\widetilde{a}_{i}^{\dagger}=u(\beta) \widetilde{a}_{i}^{\dagger}(\beta)+v(\beta) a_{i}(\beta)
\end{array} ;\right.
\end{gathered}
$$

que acarreta em,

$$
\begin{aligned}
a_{i}(\beta)|0(\beta)\rangle & =0 \\
a_{i}^{\dagger}(\beta)|0(\beta)\rangle & =\frac{a_{i}^{\dagger}}{u_{i}(\beta)}|0(\beta)\rangle .
\end{aligned}
$$

Concluiremos essa subseção calculando o valor médio do operador número de ocupação,

$$
\begin{aligned}
<N>_{\beta}= & \langle 0(\beta)|N| 0(\beta)\rangle \\
= & \left\langle 0(\beta)\left|\sum_{i=1}^{2} a_{i}^{\dagger} a_{i}\right| 0(\beta)\right\rangle, \\
= & \langle 0(\beta)| \sum_{i=1}^{2}\left(u_{i}(\beta) a_{i}^{\dagger}(\beta)+v_{i}(\beta) \widetilde{a}_{i}(\beta)\right) \times \\
& \times\left(u_{i}(\beta) a_{i}(\beta)+v_{i}(\beta) \widetilde{a}_{i}^{\dagger}(\beta)\right)|0(\beta)\rangle, \\
= & \sum_{i=1}^{2} \frac{1}{e^{\beta \omega_{i}}-1}=\sum_{i=1}^{2} v_{i}(\beta)^{2},
\end{aligned}
$$


que é justamente a distribuição de BoseEinstein associada a cada modo do oscilador harmônico bosônico, que também representare$\operatorname{mos}$ por $\overline{n_{i}}(\beta)=\frac{1}{e^{\beta \omega_{i}}-1}$.

\section{Operador Densidade Termalizado}

Nesta subseção vamos analisar a relação entre o vácuo térmico e o operador densidade, introduzindo o conceito de operador densidade termalizado associado a um estado térmico $|\Psi(\beta)\rangle$. Partindo da definição de vácuo térmico

$$
\begin{aligned}
|0(\beta)\rangle & =\sum_{n} \frac{e^{-\frac{\beta E_{n}}{2}}}{\sqrt{Z(\beta)}}|n, \widetilde{n}\rangle=\sqrt{\frac{e^{-\beta H}}{Z(\beta)}} \sum_{n}|n, \widetilde{n}\rangle \\
& =\sqrt{\rho} \sum_{n}|n, \widetilde{n}\rangle,
\end{aligned}
$$

onde $\rho=e^{-\beta H} / Z(\beta)$, a média térmica de um observável $O$ assume a forma

$$
\begin{aligned}
& \langle 0(\beta)|O| 0(\beta)\rangle=\sum_{n, m}\langle n, \widetilde{n}|\sqrt{\rho} O \sqrt{\rho}| m, \widetilde{m}\rangle \\
& =\sum_{n, m}\langle n|\sqrt{\rho} O \sqrt{\rho}| m\rangle \delta_{n, m}=\operatorname{Tr}(\rho O) .
\end{aligned}
$$

quer $|\Psi(\beta)\rangle=f\left(a(\beta), a^{\dagger}(\beta)\right)|0(\beta)\rangle=$ $f\left(a, a^{\dagger} ; \beta\right)|0(\beta)\rangle$, a média de um observável sobre esse estado térmico é dada simultaneamente pelo valor esperado, assim como pelo traço do operador densidade térmico, isto é,

$$
\begin{aligned}
& \langle\Psi(\beta)|O| \Psi(\beta)\rangle \\
= & \operatorname{Tr}(\rho|\Psi(\beta)\rangle \\
= & \left\langle 0(\beta)\left|f^{\dagger}\left(a, a^{\dagger} ; \beta\right) O f\left(a, a^{\dagger} ; \beta\right)\right| 0(\beta)\right\rangle \\
= & \operatorname{Tr}\left(f^{\dagger}\left(a, a^{\dagger} ; \beta\right) O f\left(a, a^{\dagger} ; \beta\right) \rho\right) \\
= & \operatorname{Tr}\left(O f\left(a, a^{\dagger} ; \beta\right) \rho f^{\dagger}\left(a, a^{\dagger} ; \beta\right)\right),
\end{aligned}
$$

ou seja,

$$
\rho_{|\Psi(\beta)\rangle}=f\left(a, a^{\dagger} ; \beta\right) \rho f^{\dagger}\left(a, a^{\dagger} ; \beta\right),
$$

que é denominado de operador densidade térmico, associado ao estado $|\Psi(\beta)\rangle$.

Finalizamos essa seção apresentando o operador densidade do Oscilador Harmônico Bosônico, em termos do número de ocupação, que será utilizado ao longo do trabalho. A partir da definição do operador densidade:

$$
\rho=\frac{e^{-\beta H}}{Z(\beta)},
$$

alcançamos o resultado a seguir,

$$
\begin{aligned}
& \exp \left(-\beta \omega_{1} a_{1}^{\dagger} a_{1}-\beta \omega_{2} a_{2}^{\dagger} a_{2}\right)=\sum_{n_{1}, n_{2}=0}^{\infty} \exp \left(-\beta \omega_{1} a_{1}^{\dagger} a_{1}-\beta \omega_{2} a_{2}^{\dagger} a_{2}\right)\left|n_{1} n_{2}\right\rangle\left\langle n_{1} n_{2}\right|, \\
= & \sum_{n_{1}, n_{2}=0}^{\infty}\left[1+\left(-\beta \omega_{1} a_{1}^{\dagger} a_{1}-\beta \omega_{2} a_{2}^{\dagger} a_{2}\right)+\frac{\left(-\beta \omega_{1} a_{1}^{\dagger} a_{1}-\beta \omega_{2} a_{2}^{\dagger} a_{2}\right)^{2}}{2}+\ldots\right]\left|n_{1} n_{2}\right\rangle\left\langle n_{1} n_{2}\right|, \\
= & \sum_{n_{1}, n_{2}=0}^{\infty} 1\left|n_{1} n_{2}\right\rangle\left\langle n_{1} n_{2}\left|+\left(-\beta \omega_{1} a_{1}^{\dagger} a_{1}-\beta \omega_{2} a_{2}^{\dagger} a_{2}\right)\right| n_{1} n_{2}\right\rangle\left\langle n_{1} n_{2}\right| \\
& +\frac{1}{2}\left(-\beta \omega_{1} a_{1}^{\dagger} a_{1}-\beta \omega_{2} a_{2}^{\dagger} a_{2}\right)^{2}\left|n_{1} n_{2}\right\rangle\left\langle n_{1} n_{2}\right|+\ldots, \\
= & \sum_{n_{1}, n_{2}=0}^{\infty} 1\left|n_{1} n_{2}\right\rangle\left\langle n_{1} n_{2}\left|+\left(-\beta \omega_{1} n_{1}-\beta \omega_{2} n_{2}\right)\right| n_{1} n_{2}\right\rangle\left\langle n_{1} n_{2}\right| \\
& +\frac{1}{2}\left(-\beta \omega_{1} a_{1}^{\dagger} a_{1}-\beta \omega_{2} a_{2}^{\dagger} a_{2}\right)\left(-\beta \omega_{1} n_{1}-\beta \omega_{2} n_{2}\right)\left|n_{1} n_{2}\right\rangle\left\langle n_{1} n_{2}\right|+\ldots
\end{aligned}
$$


e efetuando as simplificações obtemos,

$$
\begin{aligned}
& \sum_{n_{1}, n_{2}=0}^{\infty}\left[1+\left(-\beta \omega_{1} n_{1}-\beta \omega_{2} n_{2}\right)+\frac{\left(-\beta \omega_{1} n_{1}-\beta \omega_{2} n_{2}\right)^{2}}{2}+\ldots\right]\left|n_{1} n_{2}\right\rangle\left\langle n_{1} n_{2}\right|, \\
= & \sum_{n_{1}, n_{2}=0}^{\infty} \exp \left(-\beta \omega_{1} n_{1}\right) \exp \left(-\beta \omega_{2} n_{2}\right)\left|n_{1} n_{2}\right\rangle\left\langle n_{1} n_{2}\right|, \\
= & \sum_{n_{1}, n_{2}=0}^{\infty}\left(\frac{\overline{n_{1}}(\beta)}{1+\overline{n_{1}}(\beta)}\right)^{n_{1}}\left(\frac{\overline{n_{2}}(\beta)}{1+\overline{n_{2}}(\beta)}\right)^{n_{2}}\left|n_{1} n_{2}\right\rangle\left\langle n_{1} n_{2}\right|,,
\end{aligned}
$$

onde utilizamos a relação de completeza e a representação pelo número de ocupação para dois modos, definido na subseção IIB.

A função de partição $Z(\beta)$ é dada por,

$$
\begin{aligned}
& \operatorname{Tr}\left[\sum_{n_{1}, n_{2}=0}^{\infty} \exp \left(-\beta \omega_{1} n_{1}\right) \exp \left(-\beta \omega_{2} n_{2}\right)\left|n_{1} n_{2}\right\rangle\left\langle n_{1} n_{2}\right|\right] \\
= & \sum_{n_{1}, n_{2}, n_{1}^{\prime}, n_{2}^{\prime}=0}^{\infty} \exp \left(-\beta \omega_{1} n_{1}\right) \exp \left(-\beta \omega_{2} n_{2}\right)=\frac{1}{1-e^{-\beta \omega_{1}}} \frac{1}{1-e^{-\beta \omega_{2}}} \\
= & {\left[\frac{1}{1+\overline{n_{1}}(\beta)}\right]^{-1}\left[\frac{1}{1+\overline{n_{2}}(\beta)}\right]^{-1}, }
\end{aligned}
$$

o que resulta no operador densidade do Oscilador Harmônico Bosônico com dois modos:

$$
\rho=\frac{1}{1+\overline{n_{1}}(\beta)} \frac{1}{1+\overline{n_{2}}(\beta)} \sum_{n_{1}, n_{2}=0}^{\infty}\left(\frac{\overline{n_{1}}(\beta)}{1+\overline{n_{1}}(\beta)}\right)^{n_{1}}\left(\frac{\overline{n_{2}}(\beta)}{1+\overline{n_{2}}(\beta)}\right)^{n_{2}}\left|n_{1} n_{2}\right\rangle\left\langle n_{1} n_{2}\right| .
$$

\section{PURIFICAÇÃO E DINÂMICA DE CAMPOS TÉRMICOS}

O procedimento de purificação corresponde a uma técnica operacional, intimamente ligada à decomposição de Schmidt, que possibilita, tal qual na dinâmica de campos térmicos, relacionar estados puros com estados mistos. Temos como objetivo exibir uma certa "equivalência " entre estes dois procedimentos. Apresentaremos processo de purificação tal como o descrito na referência [6]. Considere então a seguinte decomposição ortonormal de um estado misto $\rho^{A}$ :

$$
\rho^{A}=\sum_{i} p_{i}\left|i^{A}\right\rangle\left\langle i^{A}\right|
$$

Podemos introduzir um sistema fictício $F$, que possui o mesmo espaço de estados que $A$, com uma base ortonormal $\left|i^{F}\right\rangle$. Então, um estado puro para o sistema $A F$ é dado por,

$$
|A F\rangle=\sum_{i} \sqrt{p_{i}}\left|i^{A}\right\rangle\left|i^{F}\right\rangle,
$$


com o respectivo dual,

$$
\langle A F|=\sum_{j} \sqrt{p_{j}}\left\langle j^{F}\right|\left\langle j^{A}\right| .
$$

Calculando o operador densidade reduzido do sistema $A$, partindo de $|A F\rangle$, temos:

$$
\begin{aligned}
& \operatorname{Tr}_{F}(|A F\rangle\langle A F|) \\
= & \operatorname{Tr}_{F}\left(\sum_{i, j} \sqrt{p_{i}} \sqrt{p_{j}}\left|i^{A}\right\rangle\left|i^{F}\right\rangle\left\langle j^{F}\right|\left\langle j^{A}\right|\right) \\
= & \sum_{i, j} \sqrt{p_{i} p_{j}}\left|i^{A}\right\rangle\left\langle j^{A}\right| \operatorname{Tr}_{F}\left(\left|i^{F}\right\rangle\left\langle j^{F}\right|\right) \\
= & \sum_{i, j} \sqrt{p_{i} p_{j}}\left|i^{A}\right\rangle\left\langle j^{A}\right| \delta_{i j} \\
= & \sum_{i} p_{i}\left|i^{A}\right\rangle\left\langle i^{A}\right|=\rho^{A}
\end{aligned}
$$

ou seja, o operador densidade reduzido do sistema $A$, partindo de $|A F\rangle$,coincide com $\rho^{A}$. Portanto, o estado puro é um estado cuja base de Schmidt corresponde à base formada a partir dos estados ortonormais do sistema $A$.

$\mathrm{Na}$ dinâmica de campos térmicos, por sua vez, um estado puro associado a uma mistura estatística é construído a partir da igualdade,

$$
\langle\Psi(\beta)|O| \Psi(\beta)\rangle=\operatorname{Tr}\left(\rho_{|\Psi(\beta)\rangle} O\right) .
$$

Podemos verificar se o estado purificado $|A F\rangle$, definido anteriormente também satisfaz esta igualdade. De fato,

$$
\begin{aligned}
& \langle A F|O| A F\rangle \\
= & \sum_{i, j} \sqrt{p_{i} p_{j}}\left\langle j^{F}\left|\left\langle j^{A}|(O \otimes 1)| i^{A}\right\rangle\right| i^{F}\right\rangle \\
= & \sum_{i, j} \sqrt{p_{i} p_{j}}\left\langle j^{F} \mid i^{F}\right\rangle\left\langle j^{A}|O| i^{A}\right\rangle \\
= & \sum_{i} p_{i}\left\langle i^{A}|O| i^{A}\right\rangle \\
= & \sum_{i} p_{i} \sum_{n}\left\langle n|O| i^{A}\right\rangle\left\langle i^{A} \mid n\right\rangle \\
= & \sum_{i} p_{i} \operatorname{Tr}\left(O\left|i^{A}\right\rangle\left\langle i^{A}\right|\right) \\
= & \operatorname{Tr}\left(\sum_{i} p_{i}\left|i^{A}\right\rangle\left\langle i^{A}\right| O\right)=\operatorname{Tr}\left(\rho^{A} O\right),
\end{aligned}
$$

onde utilizamos a novamente a relação de com- pleteza.

Por conseguinte, a "equivalência" está verificada. Essa é uma construção alternativa de um estado puro correspondente a uma certa matriz densidade que reproduz os mesmos valores esperados para os observáveis quânticos. À guisa de exemplo, considere a seguinte mistura estatística,

$$
\rho_{|n(\beta)\rangle}=k^{n+1} \sum_{r=0}^{\infty} k_{1}^{r} \frac{(n+r) !}{n ! r !}|n+r\rangle\langle n+r| .
$$

em que $k=\frac{1}{1+\bar{n}(\beta)}$ e $k_{1}=\frac{\bar{n}(\beta)}{1+\bar{n}(\beta)}$.

Temos que o estado térmico $|n(\beta)\rangle$, relacionado a este estado misto, é dado por,

$$
|n(\beta)\rangle=f\left(a, a^{\dagger}, \beta\right)|0(\beta)\rangle .
$$

Portanto $\rho_{|n(\beta)\rangle}$ pode ser escrito como:

$$
\rho_{|n(\beta)\rangle}=\frac{1}{n !} \frac{1}{[u(\beta)]^{2 n}}\left(a^{\dagger}\right)^{n} k \sum_{r=0}^{\infty} k_{1}^{r}|r\rangle\langle r| a^{n},
$$

e consequentemente,

$$
f\left(a, a^{\dagger}, \beta\right)=\frac{1}{\sqrt{n !}[u(\beta)]^{n}}\left(a^{\dagger}\right)^{n},
$$

de modo que o estado térmico relacionado a $\rho_{|n(\beta)\rangle}$ é dado por,

$$
\begin{aligned}
|n(\beta)\rangle & =\frac{1}{\sqrt{n !}}\left[a^{\dagger}(\beta)\right]^{n}|0(\beta)\rangle \\
& =\frac{1}{\sqrt{n !}[u(\beta)]^{n}}\left(a^{\dagger}\right)^{n}|0(\beta)\rangle \\
& =\frac{1}{\sqrt{n !}[u(\beta)]^{n}}\left(a^{\dagger}\right)^{n}\left(\sum_{r=0}^{\infty} e^{-\beta \omega r / 2}|r, \widetilde{r}\rangle\right) .
\end{aligned}
$$

Por outro lado, utilizando a decomposição de Schmidt, temos:

$$
\left|n^{\prime}(\beta)\right\rangle=\sum_{r=0}^{\infty} \sqrt{k_{1}^{n+1} k_{2} \frac{(n+r) !}{n ! r !}}|n+r, n+r\rangle .
$$

Nitidamente as expressões (44) e (45) são distintas. Malgrado este fato, produzem os mesmos valores médios para os observáveis. 
Vale destacar que a dinâmica de campos térmicos é um formalismo geral de grande aplicabilidade enquanto que a purificação é apenas um procedimento operacional simples e de amplo interesse para a computação quântica e informação quântica. Nosso objetivo nessa seção foi apenas aludir a similitude entre ambas construções.

\section{TELETRANSPORTE QUÂNTICO DE ESTADOS TÉRMICOS}

Consideremos então um protocolo de teletransporte quântico térmico em que o estado a ser teletransportado está a uma temperatura nula e o canal quântico compartilhado entre a emissora, Maria, e o receptor, João, está à temperatura finita. Deseja-se teletransportar o estado,

$$
|\Psi\rangle_{L}=x|0\rangle_{L}+y|1\rangle_{L}
$$

onde o subíndice $L$ denota $q$-bits abstratos, sem uma representação física particular.

Considere que João e Maria compartilhem um estado térmico emaranhado do tipo Bell:

$$
\left|\digamma^{+}(\beta)\right\rangle_{L}=\frac{1}{\sqrt{2}}\left(|0(\beta) 0(\beta)\rangle_{L}+|1(\beta) 1(\beta)\rangle_{L}\right),
$$

por conseguinte, o estado do sistema total corresponde ao produto tensorial do estado a ser teletransportado, dado pela Eq. (46), e o estado de Bell térmico compartilhado,

$$
\begin{aligned}
\left|\Psi_{s}\right\rangle_{L}= & |\Psi\rangle_{L} \otimes\left|\digamma^{+}(\beta)\right\rangle_{L} \\
& =\left[x|0\rangle_{L}+y|1\rangle_{L}\right] \otimes \frac{1}{\sqrt{2}}\left(|0(\beta) 0(\beta)\rangle_{L}\right. \\
& +|1(\beta) 1(\beta)\rangle)_{L} .
\end{aligned}
$$

Podemos reescrever o estado $\left|\Psi_{s}\right\rangle_{L}$ seguindo a prescrição [8], explicitando os estados de Bell térmicos,

$$
\begin{aligned}
\left|\Psi_{s}\right\rangle_{L}= & \frac{1}{\sqrt{2}}\left\{x|0\rangle_{L}|0(\beta) 0(\beta)\rangle_{L}+x|0\rangle_{L}|1(\beta) 1(\beta)\rangle_{L}+y|1\rangle_{L}|0(\beta) 0(\beta)\rangle_{L}+y|1\rangle_{L}|1(\beta) 1(\beta)\rangle_{L}\right\} \\
= & \frac{1}{2}\left\{x\left[\left|\Phi^{+}(\beta)\right\rangle_{L}+\left|\Phi^{-}(\beta)\right\rangle_{L}\right]|0(\beta)\rangle_{L}+x\left[\left|\Psi^{+}(\beta)\right\rangle_{L}+\left|\Psi^{-}(\beta)\right\rangle_{L}\right]|1(\beta)\rangle_{L}\right. \\
& \left.+y\left[\left|\Psi^{+}(\beta)\right\rangle_{L}-\left|\Psi^{-}(\beta)\right\rangle_{L}\right]|0(\beta)\rangle_{L}+y\left[\left|\Phi^{+}(\beta)\right\rangle_{L}-\left|\Phi^{-}(\beta)\right\rangle_{L}\right]|1(\beta)\rangle_{L}\right\} \\
= & \frac{1}{2}\left\{\left|\Phi^{+}(\beta)\right\rangle_{L}\left(x|0(\beta)\rangle_{L}+y|1(\beta)\rangle_{L}\right)+\left|\Phi^{-}(\beta)\right\rangle_{L}\left(x|0(\beta)\rangle_{L}-y|1(\beta)\rangle_{L}\right)\right. \\
& \left.+\left|\Psi^{+}(\beta)\right\rangle_{L}\left(y|0(\beta)\rangle_{L}+x|1(\beta)\rangle_{L}\right)+\left|\Psi^{-}(\beta)\right\rangle_{L}\left(-y|0(\beta)\rangle_{L}+x|1(\beta)\rangle_{L}\right)\right\}
\end{aligned}
$$

onde

$$
\begin{aligned}
\left|\Phi^{+}(\beta)\right\rangle_{L} & =\frac{1}{\sqrt{2}}\left(|00(\beta)\rangle_{L}+|11(\beta)\rangle_{L}\right), \\
\left|\Phi^{-}(\beta)\right\rangle_{L} & =\frac{1}{\sqrt{2}}\left(|00(\beta)\rangle_{L}-|11(\beta)\rangle_{L}\right), \\
\left|\Psi^{+}(\beta)\right\rangle_{L} & =\frac{1}{\sqrt{2}}\left(|01(\beta)\rangle_{L}+|10(\beta)\rangle_{L}\right), \\
\left|\Psi^{-}(\beta)\right\rangle_{L} & =\frac{1}{\sqrt{2}}\left(|01(\beta)\rangle_{L}-|10(\beta)\rangle_{L}\right),
\end{aligned}
$$

correspondem à estados térmicos do tipo Bell, onde a termalização só ocorre no $2^{\circ}$ modo. No limite para $T$ tendendo zero recuperamos o estado de Bell não termalizado.

Como mostrado na Eq. (49) temos quatro possibilidades de resultados para o $q$-bit de João, condicionados ao resultados das medições de Maria, ou seja,

$$
\begin{aligned}
\left|\Phi^{+}(\beta)\right\rangle_{L} \rightarrow & x|0(\beta)\rangle_{L}+y|1(\beta)\rangle_{L} \\
& =I|\Psi(\beta)\rangle_{L}, \\
\left|\Psi^{+}(\beta)\right\rangle_{L} \rightarrow & y|0(\beta)\rangle_{L}+x|1(\beta)\rangle_{L} \\
& =X|\Psi(\beta)\rangle_{L},
\end{aligned}
$$




$$
\begin{aligned}
\left|\Phi^{-}(\beta)\right\rangle_{L} \rightarrow & x|0(\beta)\rangle_{L}-y|1(\beta)\rangle_{L} \\
& =Z|\Psi(\beta)\rangle_{L}, \\
\left|\Psi^{-}(\beta)\right\rangle_{L} \rightarrow & -y|0(\beta)\rangle_{L}+x|1(\beta)\rangle_{L} \\
& =X Z|\Psi(\beta)\rangle_{L} .
\end{aligned}
$$

Ao realizar sua medida, Maria deve comunicar o seu resultado a João, por algum canal clássico, que de posse do resultado deverá executar uma operação unitária, como as descritas na Eq. (3), "recuperando " o estado original.

Se o resultado de Maria for $\left|\Phi^{+}(\beta)\right\rangle_{L}$, nada deve ser feito ao estado de João; se for $\left|\Psi^{+}(\beta)\right\rangle_{L}$, a porta lógica $X$ deverá ser aplicada; caso o resultado seja $\left|\Phi^{-}(\beta)\right\rangle_{L}$, a porta lógica necessária será $Z$ e se o resultado for $\left|\Psi^{-}(\beta)\right\rangle_{L}$, devemos aplicar $Z X$, já que $Z X\left\{-y|0(\beta)\rangle_{L}+x|1(\beta)\rangle_{L}\right\}=$ $Z X\left[X Z|\Psi(\beta)\rangle_{L}\right]=|\Psi(\beta)\rangle_{L}$

Encerramos está subseção apresentando o Teorema da Não-Clonagem, de capital importância para o teletransporte quântico, proposto em 1982 [9, 10] e para o qual já existe uma série de desenvolvimentos posteriores. [11 14]. O teorema da não-clonagem afirma que podemos copiar a informação clássica de forma exata ou aproximada, contudo a mecânica quântica proíbe a cópia de estados quânticos desconhecidos.

Suponhamos então uma máquina quântica equipada com dois compartimentos A e B. O compartimento A é denominado compartimento de dados e o compartimento B, compartimento-alvo. Vamos considerar também que o compartimento A esteja inicialmente em um estado desconhecido $|\Psi(\beta)\rangle$ e o compartimento B em um estado $|s(\beta)\rangle$. Então, o estado inicial da máquina de clonagem é dado por,

$$
|\Psi s(\beta)\rangle=|\Psi(\beta)\rangle \otimes|s(\beta)\rangle .
$$

Suponha que podemos implementar o procedimento de cópia através de uma operação unitária, $U$, copiando o estado desconhecido $|\Psi(\beta)\rangle$,

$$
\begin{aligned}
|\Psi(\beta)\rangle \otimes|s(\beta)\rangle \rightarrow & U(|\Psi(\beta)\rangle \otimes|s(\beta)\rangle) \\
& =|\Psi(\beta)\rangle \otimes|\Psi(\beta)\rangle .
\end{aligned}
$$

Se realizarmos cópias de dois estados, $|\Psi(\beta)\rangle$ e $|\Phi(\beta)\rangle$ :

$$
\begin{aligned}
& U(|\Psi(\beta)\rangle \otimes|s(\beta)\rangle)=|\Psi(\beta)\rangle \otimes|\Psi(\beta)\rangle, \\
& U(|\Phi(\beta)\rangle \otimes|s(\beta)\rangle)=|\Phi(\beta)\rangle \otimes|\Phi(\beta)\rangle,
\end{aligned}
$$

o produto interno das equações (55) e (56) resulta em,

$$
\begin{aligned}
& \left(\left\langles(\beta)|\otimes\langle\Psi(\beta)|) U^{\dagger} U(|\Phi(\beta)\rangle \otimes|s(\beta)\rangle)\right.\right. \\
& =(\langle\Psi(\beta)|\otimes\langle\Psi(\beta)|)(|\Phi(\beta)\rangle \otimes|\Phi(\beta)\rangle), \\
& \langle s(\beta) \mid s(\beta)\rangle\langle\Psi(\beta) \mid \Phi(\beta)\rangle=(\langle\Psi(\beta) \mid \Phi(\beta)\rangle)^{2}, \\
& \langle\Psi(\beta) \mid \Phi(\beta)\rangle=(\langle\Psi(\beta) \mid \Phi(\beta)\rangle)^{2} .
\end{aligned}
$$

Temos somente duas soluções: $|\Psi(\beta)\rangle=$ $|\Phi(\beta)\rangle$ ou $|\Psi(\beta)\rangle \perp|\Phi(\beta)\rangle$, ou os estados são iguais ou são ortogonais. Consequentemente, tal máquina de clonagem pode copiar apenas estados ortogonais entre si.

\section{A. Representação Duplo-Trilho e Álgebra de Lie $s u(2)$}

Com a introdução da temperatura, via o formalismo DCT, o estado térmico "recuperado", nas relações (51) e (52), pode não corresponder, necessariamente, ao estado original devido aos efeitos da temperatura, pois a faixa térmica em que se encontra o estado é capaz de alterar as suas características. Nosso objetivo é investigar o quão distante do estado original, o estado final se encontra. Para isso representaremoz os $q$-bits em termos de estados de Fock, usando a representação duplo-trilho:

$$
\begin{aligned}
|0\rangle_{L} & \rightarrow|01\rangle, \\
|1\rangle_{L} & \rightarrow|10\rangle .
\end{aligned}
$$

A representação duplo-trilho permite a aplicação de portas lógicas quânticas utilizando deslocadores de fase, divisores de feixe e meios Kerr não linear, além de simplificar a forma de correção de erros e tolerar mais a decorerência [15, 16].

Condicionado ao resultado das medições de Maria, como apresentado na Eq. (51) e (52), teremos o estado $|\Psi(\beta)\rangle_{L}$ teletransportado a 
João, que deverá atuar uma das portas lógicas quânticas já discutidas. Em consonância com a associação supradita, iremos representar o estado $|\Psi(\beta)\rangle_{L}$ na representação duplo-trilho e associar as portas lógicas quânticas a operadores de uma álgebra de Lie $s u(2)$, atuando neste espaço. Portanto $|\Psi\rangle=x|01\rangle+y|10\rangle \mathrm{e}$,

$$
\begin{aligned}
|\Psi(\beta)\rangle & =x|0(\beta) 1(\beta)\rangle+y|1(\beta) 0(\beta)\rangle \\
& \equiv x|\beta, 01 \widetilde{00}\rangle+y|\beta, 1000\rangle,
\end{aligned}
$$

onde

$$
\begin{aligned}
|0(\beta) 1(\beta)\rangle= & |\beta, 01 \widetilde{00}\rangle=a_{2}^{\dagger}(\beta)|\beta, 00 \widetilde{00}\rangle \\
& =a_{2}^{\dagger}(\beta)|0(\beta) 0(\beta)\rangle, \\
|1(\beta) 0(\beta)\rangle= & |\beta, 10 \widetilde{00}\rangle=a_{1}^{\dagger}(\beta)|\beta, 00 \widetilde{00}\rangle \\
& =a_{1}^{\dagger}(\beta)|0(\beta) 0(\beta)\rangle .
\end{aligned}
$$

Rememoremos que as matrizes de Pauli $X, Y$ e $Z$ geram o grupo $S U(2)$, assim como os operadores $S_{+}, S_{-}$e $S_{0}$ que atuando no Oscilador Harmônico com dois modos [6, 7],

$$
\begin{aligned}
& \left\{\begin{array}{l}
S_{+}=a_{1}^{\dagger} a_{2} \\
S_{-}=a_{2}^{\dagger} a_{1} \\
S_{0}=a_{1}^{\dagger} a_{1}-a_{2}^{\dagger} a_{2}
\end{array}\right. \\
& \left\{\begin{array}{r}
X \leftrightarrow S_{+}+S_{-} \\
Y \leftrightarrow-i\left(S_{+}-S_{-}\right) \\
Z \leftrightarrow S_{0} .
\end{array}\right.
\end{aligned}
$$

Aplicando a transformação de Bougoliubov na Eq. 63 obtemos os operadores: termalizados,

$$
\begin{aligned}
S_{+}(\beta) & =a_{1}^{\dagger}(\beta) a_{2}(\beta) \quad S_{-}(\beta)=a_{2}^{\dagger}(\beta) a_{1}(\beta), \\
S_{0}(\beta) & =a_{1}^{\dagger}(\beta) a_{1}(\beta)-a_{2}^{\dagger}(\beta) a_{2}(\beta),
\end{aligned}
$$

que atende a álgebra de Lie do grupo $S U(2)$,

$$
\begin{aligned}
{\left[S_{+}(\beta), S_{-}(\beta)\right] } & =S_{0}(\beta) \\
{\left[S_{0}(\beta), S_{ \pm}(\beta)\right] } & = \pm S_{ \pm}(\beta),
\end{aligned}
$$

com,

$$
\begin{aligned}
& S_{+}(\beta)\left|n_{1}(\beta), n_{2}(\beta)\right\rangle \\
& =\sqrt{n_{2}\left(n_{1}+1\right)}\left|\left(n_{1}+1\right)(\beta),\left(n_{2}+1\right)(\beta)\right\rangle,
\end{aligned}
$$

e

$$
\begin{aligned}
& S_{-}(\beta)\left|n_{1}(\beta), n_{2}(\beta)\right\rangle \\
& =\sqrt{n_{1}\left(n_{2}+1\right)}\left|\left(n_{1}-1\right)(\beta),\left(n_{2}+1\right)(\beta)\right\rangle, \\
& S_{0}(\beta)\left|n_{1}(\beta), n_{2}(\beta)\right\rangle \\
& =\left(n_{1}-n_{2}\right)\left|n_{1}(\beta), n_{2}(\beta)\right\rangle .
\end{aligned}
$$

Como $X \equiv a_{1}^{\dagger}(\beta) a_{2}(\beta)+a_{2}^{\dagger}(\beta) a_{1}(\beta)$ e $Z \equiv$ $a_{1}^{\dagger}(\beta) a_{1}(\beta)-a_{2}^{\dagger}(\beta) a_{2}(\beta)$, as portas lógicas quânticas, necessárias para a recuperação do estado teletransportado, podem ser obtidas por:

$$
\begin{aligned}
& X|0(\beta)\rangle_{L} \leftrightarrow\left[S_{+}(\beta)+S_{-}\right](\beta)|0(\beta) 1(\beta)\rangle \\
& =|1(\beta) 0(\beta)\rangle, \\
& X|1(\beta)\rangle_{L} \leftrightarrow\left[S_{+}(\beta)+S_{-}\right](\beta)|1(\beta) 0(\beta)\rangle \\
& =|0(\beta) 1(\beta)\rangle, \\
& Z|0(\beta)\rangle_{L} \leftrightarrow S_{0}|1(\beta) 0(\beta)\rangle \\
& =|1(\beta) 0(\beta)\rangle, \\
& Z|1(\beta)\rangle_{L} \leftrightarrow S_{0}|0(\beta) 1(\beta)\rangle=-|0(\beta) 1(\beta)\rangle, \\
& X Z|0(\beta)\rangle_{L} \leftrightarrow\left[S_{+}(\beta)+S_{-}\right] S_{0}|1(\beta) 0(\beta)\rangle \\
& =|0(\beta) 1(\beta)\rangle, \\
& X Z|1(\beta)\rangle_{L} \leftrightarrow\left[S_{+}(\beta)+S_{-}\right] S_{0}|0(\beta) 1(\beta)\rangle \\
& =-|1(\beta) 0(\beta)\rangle,
\end{aligned}
$$

devido a representação duplo-trilho.

\section{B. Fidelidade}

Com o intuito de investigar o quão distante o estado original encontra-se do estado final termalizado, iremos calcular a Fidelidade Quântica, que é uma medida de distância entre estados do espaço de Hilbert, definida por $F=\sqrt{\langle\Psi|\rho| \Psi\rangle}$, onde $|\Psi\rangle$ é um puro e $\rho$ um estado arbitrário [6].

Como estamos interessados em analisar quanto o estado inicial se distancia de si 
mesmo, devido aos efeitos térmicos, iremos calcular a Fidelidade entre o estado $|\Psi\rangle$ e a sua versão termalizada $\rho_{|\Psi(\beta)\rangle}$, ou seja,

$$
F=\sqrt{\left\langle\Psi\left|\rho_{|\Psi(\beta)\rangle}\right| \Psi\right\rangle},
$$

tentando aferir como a preservação da informação quântica se dá com a variação da temperatura. A fidelidade varia entre 0 e 1 , onde valores próximos de 1 representam estados próximo entre si e valores próximos de 0 representam estados cada vez mais diferentes e distinguíveis.

Vamos agora calcular o operador densidade termalizado $\rho_{|\Psi(\beta)\rangle}$, e para isso utilizaremos a relação 29 a fim de reescrever o estado $|\Psi(\beta)\rangle$,

$$
\begin{aligned}
|\Psi(\beta)\rangle= & \frac{x}{u_{2}(\beta)} a_{2}^{\dagger}|0(\beta) 0(\beta)\rangle \\
& +\frac{y}{u_{1}(\beta)} a_{1}^{\dagger}|0(\beta) 0(\beta)\rangle .
\end{aligned}
$$

com respectivo dual,

$$
\begin{aligned}
\langle\Psi(\beta)|= & \langle 0(\beta) 0(\beta)| a_{2} \frac{x^{*}}{u_{2}(\beta)} \\
& +\langle 0(\beta) 0(\beta)| a_{1} \frac{y^{*}}{u_{1}(\beta)} .
\end{aligned}
$$

Utilizando a Eq. (33), o operador $\rho_{|\Psi(\beta)\rangle}$ é,

$$
\begin{aligned}
\rho_{|\Psi(\beta)\rangle}= & \frac{|x|^{2}}{u_{2}^{2}(\beta)} a_{2}^{\dagger} \rho a_{2}+\frac{x^{*} y}{u_{1}(\beta) u_{2}(\beta)} a_{1}^{\dagger} \rho a_{2} \\
& +\frac{y^{*} x}{u_{1}(\beta) u_{2}(\beta)} a_{2}^{\dagger} \rho a_{1}+\frac{|y|^{2}}{u_{1}^{2}(\beta)} a_{1}^{\dagger} \rho a_{1},
\end{aligned}
$$

com $\rho$ sendo o operador densidade do oscilador harmônico bosônico com dois modos, dado pela Eq. (35). Fazendo uso dos operadores de criação e aniquilação temos,

$$
\begin{aligned}
& \rho_{|\Psi(\beta)\rangle}=\frac{1}{1+\overline{n_{1}}(\beta)} \frac{1}{1+\overline{n_{2}}(\beta)} \sum_{n_{1}, n_{2}=0}^{+\infty}\left(\frac{\overline{n_{1}}(\beta)}{1+\overline{n_{1}}(\beta)}\right)^{n_{1}}\left(\frac{\overline{n_{2}}(\beta)}{1+\overline{n_{2}}(\beta)}\right)^{n_{2}} \\
& \times\left\{\frac{|x|^{2}}{u_{2}^{2}(\beta)}\left(n_{2}+1\right)\left|n_{1}\left(n_{2}+1\right)\right\rangle\left\langle n_{1}\left(n_{2}+1\right)\left|+\frac{x^{*} y}{u_{1}(\beta) u_{2}(\beta)} \sqrt{\left(n_{1}+1\right)\left(n_{2}+1\right)}\right|\left(n_{1}+1\right) n_{2}\right\rangle\left\langle n_{1}\left(n_{2}+1\right)\right|\right. \\
& \left.+\frac{y^{*} x}{u_{1}(\beta) u_{2}(\beta)} \sqrt{\left(n_{1}+1\right)\left(n_{2}+1\right)}\left|n_{1}\left(n_{2}+1\right)\right\rangle\left\langle\left(n_{1}+1\right) n_{2}\left|+\frac{|y|^{2}}{u_{1}^{2}(\beta)}\left(n_{1}+1\right)\right|\left(n_{1}+1\right) n_{2}\right\rangle\left\langle\left(n_{1}+1\right) n_{2}\right|\right\} .
\end{aligned}
$$

Uma vez que,

$$
\begin{aligned}
& \left\langle\Psi \mid n_{1}\left(n_{2}+1\right)\right\rangle\left\langle n_{1}\left(n_{2}+1\right) \mid \Psi\right\rangle=|x|^{2} \delta_{n_{1}, 0} \delta_{n_{2}, 0}, \\
& \left\langle\Psi \mid\left(n_{1}+1\right) n_{2}\right\rangle\left\langle n_{1}\left(n_{2}+1\right) \mid \Psi\right\rangle=x y^{*} \delta_{n_{1}, 0} \delta_{n_{2}, 0}, \\
& \left\langle\Psi \mid n_{1}\left(n_{2}+1\right)\right\rangle\left\langle\left(n_{1}+1\right) n_{2} \mid \Psi\right\rangle=x^{*} y \delta_{n_{1}, 0} \delta_{n_{2}, 0}, \\
& \left\langle\left.\Psi\left|\left(n_{1}+1 n_{2}\right\rangle\left\langle\left(n_{1}+1\right) n_{2} \mid \Psi\right\rangle=\right| y\right|^{2} \delta_{n_{1}, 0} \delta_{n_{2}, 0},\right.
\end{aligned}
$$

onde $\delta_{i, j}$ é a delta de Kronecker. A Fidelidade, tendo em conta a Eq. (70), resulta,

$$
\begin{aligned}
F & =\sqrt{\frac{1}{1+\overline{n_{1}}(\beta)} \frac{1}{1+\overline{n_{2}}(\beta)}} \times \\
& \times\left[\frac{|x|^{4}}{u_{2}^{2}(\beta)}+\frac{2|x|^{2}|y|^{2}}{u_{1}(\beta) u_{2}(\beta)}+\frac{|y|^{4}}{u_{1}^{2}(\beta)}\right]^{1 / 2} .
\end{aligned}
$$

Vale ressaltar que quando $T \rightarrow 0$ na Eq. (76), a fidelidade vale 1 , ou seja, à temperatura nula o estado é teletransportado com fidelidade máxima, preservando as suas características. Para o caso em que a frequência dos 
dois osciladores são iguais, isto é, $\omega_{1}=\omega_{2}$, a fidelidade resume-se a,

$$
\begin{aligned}
F & =\sqrt{\frac{1}{(1+\bar{n}(\beta))^{2}} \frac{\left(|x|^{2}+|y|^{2}\right)^{2}}{u^{2}(\beta)}} \\
& =\sqrt{\left(1-e^{-\beta \omega}\right)^{3}}
\end{aligned}
$$

lembrando que o estado é normalizado, ou seja, $|x|^{2}+|y|^{2}=1$.

Na figura 2 ilustramos a fidelidade obtida na Eq. (77), para frequências na ordem de $\mathrm{MHz}$ e $\mathrm{GHz}$ [17, 18. Na figura 2-(a) utilizamos uma frequência de $\omega=2 \pi M H z$, obtendo uma temperatura da ordem de milikelvin e na figura 2-(b) utilizamos uma frequência de $\omega=2 \pi G H z$, obtendo uma temperatura da ordem de decikelvin. O comportamento da fidelidade mostra como os efeitos térmicos são capazes de alterar as característica do estado original, de forma que a fidelidade diminui com o aumento da temperatura. Preservar a fidelidade de um estado quântico é uma condição essencial para que ocorra, de forma eficiente, o teletransporte quântico.

\section{CONCLUSÕES E PERSPECTIVAS}

O teletransporte quântico é um dos processos mais instigantes no campo da computação quântica pois permite a transmissão da informação sem o transporte de matéria ou energia. Sistemas realísticos envolvem o efeito da temperatura de modo que se faz necessário a análise dos protocolos à temperatura finita. Analisamos a similitude entre o procedimento de purificação e a duplicação na dinâmica de campos térmicos, ressaltando o caráter geral da teoria supradita. Analisamos também o teletransporte quântico de estados térmicos bosônicos utilizando o formalismo da dinâmica de campos térmicos. Em particular utilizamos a representação duplo-trilho como codificação para os q-bits lógicos. A álgebra de Lie térmica $s u(2)$ foi fundamental para a finalização do protocolo.

Seguidamente, investigamos a fidelidade do teletransporte, verificando que a fidelidade tende a 1 quando a temperatura tende a zero, o que corresponde ao efeito presumivelmente esperado. Além disso, calculamos a fidelidade para frequências na ordem de $\mathrm{MHz}$ e $\mathrm{GHz}$, apresentando os resultados. Como perspectivas pretendemos investigar os efeitos térmicos sobre o teletransporte com sistemas fermiônicos bem como avançar na compreensão da análise de temperatura sobre a computação quântica via dinâmica de campos térmicos.

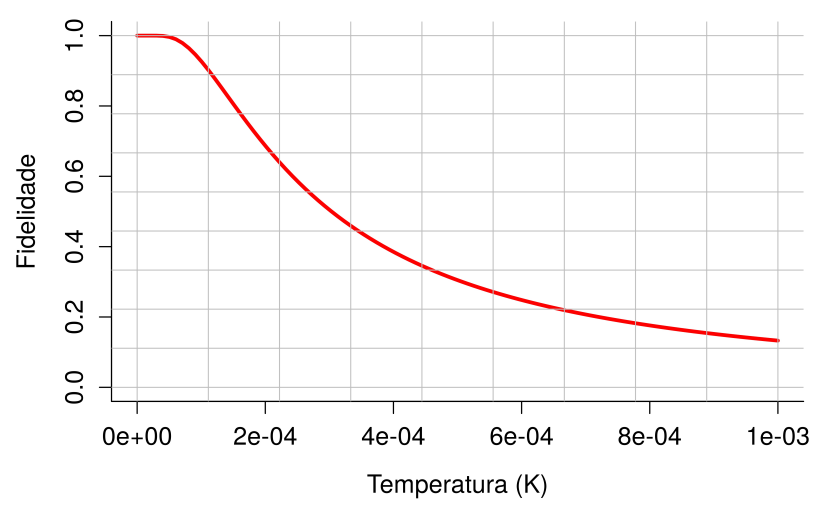

(a) Para frequência $\omega=2 \pi M H z$

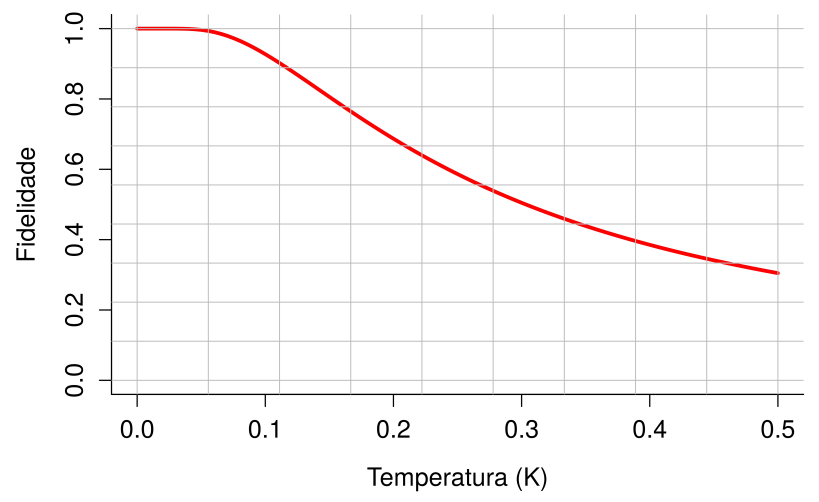

FIGURA 2: Fidelidade do estado térmico teletransportado.

(b) Para frequência $\omega=2 \pi G H z$

Fonte: Os autores. 
[1] A. Ekert, P.L. Knight, Am. J. Phys. 63, 415 (1995).

[2] C.H. Bennet, G. Brassard, C. Crépeau, R. Josza, A. Peres, W. Wooters, Phys. Rev. Lett. 70, 1895 (1993).

[3] D. Boschi, S. Branca, F. De Martini, L. Hardy, S. Popescu, Phys. Rev. Lett. 80, 1121 (1998).

[4] T.P. Oliveira, Teletransporte quântico de estados térmicos. Dissertação (Mestrado) - Instituto de Física da UnB, Brasília (2008).

[5] T.P. Trindade, Estruturas algébricas em Informação Quântica e Dinâmica de Campos Térmicos. Tese (Doutorado) - Instituto de Física da UFBA, Salvador (2010).

[6] M.A. Nielsen, I.H. Chuang, Quantum Computation and Quantum Information. Cambridge: University Press (2000).

[7] F.C. Khanna, A.P.C. Malbouisson, J.M.C. Malbouisson, A.E. Santana, Thermal Quantum Field Theory: Algebraic Aspects and Applications. Singapure: World Scientific Publishing Co. (2009).

[8] J. Preskill, Lecture Notes for Physics 229: Quantum Information and Computation (1998). Disponível em: https://www.lorentz.leidenuniv.nl/quantumcomputers/literature/preskill-_1_to_6.pdf Acessado em: 29 de janeiro de 2019.
[9] D. Dieks, Phys. Lett. A 92, 271 (1982).

[10] W.K. Wooters, W.H. Zurek, Nature 299, 802 (1982)

[11] H. Barnum, C.M. Caves, C.A. Fuchs, R. Josza, B. Schumacher, Phys. Rev. Lett. 76, 2828 (1996).

[12] T. Mor, Phys. Rev. Lett. 80, 3137 (1998).

[13] M.D. Westmoreland, B. Schumacher, arXiv:quant-ph/981014 (1998).

[14] S.J. Van Enk, arXiv: quant-ph/9805006 (1998).

[15] I.L. Chuang, Y. Yamamoto, Phys. Rev. 52, 3489 (1995).

[16] O.L.V. Rocha, Estados Quânticos do Tipo Bell na Representação Duplo-Trilho e Efeitos da Temperatura: uma Análise usando a Dinâmica de Campos Térmicos, Dissertação (Mestrado) - Instituto de Física da UFBA, Salvador (2011).

[17] D. Paredes-Barato, C.S. Adams, Phys. Rev. Lett. 112, 040501 (2014).

[18] C. Kupchak, T. Mittiga, B. Jordaan, M. Namazi, C. Nölleke, E. Figueroa, Sci. Rep. 5, 7658 (2015).

[19] Também denominado de teleporte quântico, por alguns autores. 